\title{
Epidemics in small world networks
}

\author{
M. M. Telo da Gama and A. Nunes \\ Centro de Física Teórica e Computacional and Departamento de Física, \\ Faculdade de Ciências da Universidade de Lisboa, \\ P-1649-003 Lisboa Codex, Portugal
}

\begin{abstract}
For many infectious diseases, a small-world network on an underlying regular lattice is a suitable simplified model for the contact structure of the host population. It is well known that the contact network, described in this setting by a single parameter, the small-world parameter $p$, plays an important role both in the short term and in the long term dynamics of epidemic spread. We have studied the effect of the network structure on models of immune for life diseases and found that in addition to the reduction of the effective transmission rate, through the screening of infectives, spatial correlations may strongly enhance the stochastic fluctuations. As a consequence, time series of unforced Susceptible-Exposed-Infected-Recovered (SEIR) models provide patterns of recurrent epidemics with realistic amplitudes, suggesting that these models together with complex networks of contacts are the key ingredients to describe the prevaccination dynamical patterns of diseases such as measles and pertussis. We have also studied the role of the host contact strucuture in pathogen antigenic variation, through its effect on the final outcome of an invasion by a viral strain of a population where a very similar virus is endemic. Similar viral strains are modelled by the same infection and reinfection parameters, and by a given degree of cross immunity that represents the antigenic distance between the competing strains. We have found, somewhat surprisingly, that clustering on the network decreases the potential to sustain pathogen diversity.

PACS numbers: 89.75.-k, 87.10.+c, 87.23.-n
\end{abstract}




\section{INTRODUCTION}

Nearly a decade ago, Watts and Strogatz introduced a class of networks with a topology interpolating between that of lattices and random graphs [1]. In these models a fraction of the links of the lattice is randomized by connecting nodes, with probability $p$, with any other node. For a range of $p$ the network exhibits 'small world' behaviour, where a local neighbourhood (as in lattices) coexists with a short average path length (as in random graphs). Analysis of real networks [2] reveals the existence of small worlds in many interaction networks, including networks of social contacts. Recently, attention has been focussed on the impact of network topology on the dynamics of the processes running on it with emphasis on the spreading of infectious diseases. Early numerical and analytical studies have focussed on the calculation of epidemic thresholds [4, 5] for different classes of networks and on the short term dynamics of epidemic bursts [6] for small world networks of the Watts and Strogatz type. By contrast, the effects of network topology on the long term dynamics of epidemic spread has received much less attention.

In the last couple of years we have studied simple epidemic models on dynamic (or annealed) Watts and Strogatz small world networks [7] and found that, for a large range of $p$, epidemics persist for times that approach those of homogeneously mixed populations providing suitable models to investigate the effects of spatial correlations on the long term dynamics of epidemic spread. In our model the $N$ nodes of the network represent individuals. The links are then connections along which the infection spreads. If all the links are local (lattice) the persistence of the epidemic is low and the endemic state may not be reached 6]. On the other hand, if the network is random (homogeneously mixed population), the persistence is high but the effect of spatial correlations is neglected [8]. The dynamic version of the Watts and Strogatz model [7] is thus the simplest model of a real network of social contacts [2].

Using a SEIR (Susceptible-Exposed-Infective-Recovered) model, we have obtained a quantitative description of the incidence oscillations that characterize the prevaccination recurrent epidemics of immune for life diseases such as measles and pertussis. The model, 
on the same network, was found to describe the qualitative differences observed in the long term dynamics of such diseases. We have also used a simple two-strain version of a recently proposed SIR model with reinfection to determine the outcome of an invasion in multiple strain infections. The results uncovered a surprising effect of the network structure on the diversity of pathogens, that was found to decrease, rather than increase, as the spatial correlations on the dynamic small world network increase.

\section{RECURRENT EPIDEMICS ON NETWORKS}

The Susceptible-Infected-Recovered (SIR) mean field model in a closed population of $N$ individuals is given by the equations

$$
\begin{aligned}
& \dot{S}=-\beta S I / N \\
& \dot{I}=\beta S I / N-\gamma I
\end{aligned}
$$

where $S$ (respectively $I, R$ ) is the number of susceptible (respectively infected, recovered) individuals and $\beta$ is the transmissibility. This is a model for the dynamics of a disease that confers permanent immunity and it is easily generalized to an epidemic that takes place on a network. As for models of epidemic spread on regular lattices [9], the model may be mapped onto percolation on the same network [4]. The percolation transition corresponds to the epidemic threshold, above which an epidemic outbreak is possible (i.e. one that infects a non-zero fraction of the population, in the limit of large populations) and the size of the percolating cluster above this transition corresponds to the size of the epidemic. The SEIR model is a generalisation of (11) that takes into account a latency period, during which individuals carry the pathogen but are not yet infective. This was found to be relevant in the modelling of childhood diseases [3].

The endemic persistence of a disease on a closed population requires renewal of susceptibles through demography or loss of immunity. To assess the effect of network topology on the long term dynamics of simple spatial models for childhood diseases we implemented discrete SIR and SEIR models with births and deaths corresponding to an average life time of 61 
years [7, 10, 11] on a square lattice with $N$ sites and dynamic small world interaction rules. We found characteristic medium and long-term dynamics related, in a quantitative fashion, to the structure of the network of contacts. In particular, as the small world parameter, $p$, decreases, the increase in spatial correlations (i) decreases the effective transmissibility through the screening of infectives and susceptibles, which in turn increases the value of the transmissibility at the endemic threshold. In addition, the spatial correlations (ii) enhance the stochastic fluctuations with respect to the homogeneously mixed stochastic model. This effect is particularly strong at low $p$, where the relative fluctuations are largest and where as a consequence (iii) the dependence of the steady state densities on the effective transmissibility predicted by the mean-field equations breaks down [7].

In Figure 1(a) we have plotted the incidence time series of a SEIR model 11] with the epidemiological parameters (latency period $\tau_{l}$ and recovery time $\tau_{i}$ ) taken for measles and two different values of the small world parameter $p, p=1$ and $p=0.2$. For homogeneously mixed populations, $p=1$, the infective time series exhibits incidence oscillations with an average period of two years. However, the amplitude of these oscillations is underestimated when compared with the incidence oscillations of measles in real data for similar population sizes [12]. By contrast, in the infective time series for SEIR simulations on the network for $p=0.2$ the amplitude of the incidence peaks is shown to increase significantly, in line with the real data.

As with any unforced model, the characteristic biennial cycles of prevaccination records cannot be obtained without fine tuning of the model's parameters, transmissibility, $\beta$, and probability of long-range infection, $p$. Despite this fact, which means that, for measles, seasonality cannot be ignored [13], the reported effects of spatial correlations should also be taken into account as a key issue in explaining incidence amplitudes.

Figure 1(b) shows the incidence time series of model [11] with the epidemiological parameters taken for pertussis and three different values of the small world parameter $p, p=1$, $p=0.2$ and $p=0.1$. Again, we see that for the smaller value of $p$ the amplitude of the incidence peaks is significantly enhanced with respect to the homogeneously mixed case $p=1$. 
However, for intermediate values of $p$, and in particular for $p=0.2$, this effect is almost negligible.

The main conclusion of the previous analysis is that the impact of the spatial correlations depends also on the stability of a particular disease, measured by the distance of the endemic phase to the corresponding threshold on the network, that is disease dependent [18].

\section{EVOLUTIONARY DYNAMICS OF MULTIPLE STRAINS}

We consider the invasion by a pathogen strain of a population where another strain is endemic. A single viral strain is meant to represent a cluster of co-circulating antigenically close strains, and its uncoupled dynamics is described by a SIR model with transmissibility $\beta$ for first infection and partial immunity against reinfection. The reinfection parameter $\sigma$ of the model is the ratio between disease transmissibilities for reinfection and for first infection. It measures the size of the cluster, in terms of antigenic distance.

For realistic values of the birth rate, the SIR model with partial immunity exhibits an abrupt change in the levels of infection as $\beta$ increases across a transmissibility threshold. This region of abrupt change of infection prevalence is related to a bifurcation that occurs

in the model with zero birth rate [7], and we shall use the term reinfection threshold for the smoothed transition that takes place in models with small birth rates. Recent studies [14, 15] have revealed a connection between the reinfection threshold and the potential for pathogen diversity, that increases as the level of infection increases.

In two strain dynamics, disease transmissibility for infection by one of the strains of individuals previously infected by the other strain is also reduced by a factor $\sigma_{\times}$, due to crossimmunity. We assume that cross-immunity is weaker than within strain partial immunity, reflecting the fact that the antigenic distance between clusters must be larger than the cluster size. Therefore, we take $\sigma<\sigma_{\times}$, and $\delta=\sigma_{\times}-\sigma$ is a measure of the difference between the two competing strains, in terms of the immune response that they trigger.

For a model with two (symmetrical) strains we have found that, at the level of mean field approximation, the reinfection threshold is the boundary between two different regimes. For 
similar strains $(\delta \approx 0.02)$, the behaviour of the model below threshold is strain replacement, and the behaviour on and above threshold is strain coexistence. For distinct viral strains $(\delta \approx 0.2)$, the outcome is always strain coexistence, but the density oscillations are negligible above threshold, and very pronounced below threshold, implying that taking into account that the population is discrete, for realistic population sizes we find extinction of both strains below threshold 7]. This scenario has been confirmed by simulations of a stochastic model for homogeneously mixed populations [16]. In the following we consider the contact structure of the host population and investigate its effect on pathogen diversity and evolution.

We performed individual based simulations starting from an initial condition where the system is close to the steady state for a single resident strain and introduced a small fraction of individuals infected by the invading strain. We found that as local effects become important (low $p$ ), strain replacement (and also total extinction) are favoured with respect to the homogeneously mixed regime $(p=1)$. This happens both for similar $(\delta \approx 0.02)$ and for distinct strains $(\delta \approx 0.2)$, and, as opposed to the effect reported in the preceeding section, does not require $p$ to be close to the epidemic persistence transition, only that the competing strains have moderate transmissibilities (see Figure 2).

Our results differ from those reported in [17] for a model of strain evolution, with a high rate of supply of naive susceptibles, on a (static) small world network of contacts, where spatial correlations were found to favour pathogen diversity by reducing the spread of acquired immunity. In our model, with a low rate of supply of naive susceptibles, the main effect of spatial correlations is the screening of infectives that leads to a reduction of the effective transmissibility. As $p$ decreases, this effect brings a system above the reinfection threshold closer to or even below the reinfection threshold, also favouring replacement for similar strains and either replacement or extinction for distinct ones.

Since screening of infectives occurs quite generally in structured populations, the effect reported here should be fairly independent of the network's detailed topology and correlations. 


\section{CONCLUSIONS}

Our results for the SEIR model support the conclusion that, for some purposes, successful modeling of disease spread must take into account that populations are finite and discrete, and must include a realistic representation of the spatial degrees of freedom or, more generally, of the interaction network topology. We suggest a reassessment of the impact of environmental forcing by studying its effects on a realistic autonomous model as the one that we propose. In particular, fluctuation enhancement by spatial correlations might remove some of the constraints on the strength of seasonality and avoid fine tuning of seasonal forcing amplitudes.

In the context of multiple strain infections and the evolution and diversity of pathogens, the results of our simulations strongly support the conclusion that in systems with reinfection and low rates of supply of naive susceptibles, the major effect of the host population structuring is a reduction of pathogen diversity.

\section{ACKNOWLEDGEMENTS}

Financial support from the Portuguese Foundation for Science and Technology (FCT) under contract POCTI/ESP/44511/2002 is gratefully acknowledged. The authors also acknowledge the contributions of J. P. Torres and M. Simões to test and to improve the code used in the simulations.

[1] D. J. Watts and S. H. Strogatz, Nature 392, 440 (1998).

[2] S. Eubank, H. Guclu, V. S. Anil Kumar, M. V. Marathe, A. Srinivasan, Z. Torockzcai and N. Wang, Nature 429, 180 (2004).

[3] R. A. Anderson and R. M. May, Infectious Diseases of Humans, Oxford U. P., Oxford, 1991.

[4] C. Moore and M. E. J. Newmann, Phys. Rev. E 61, 5678 (2000); C. Moore and M. E. J. Newmann, Phys. Rev. E 62, 7059 (2000). 
[5] R. Pastor-Santorras and A. Vespigniani, Phys. Rev. Lett. 86, 3200 (2001); R. M. May and A. L. Lloyd, Phys. Rev. E 64, 066112 (2001).

[6] C. J. Rhodes and R. M. Anderson, J. Theor. Biol. 180, 125 (1996); A. Kleczkowski and B. T. Grenfell, Physica A 274, 355 (1999).

[7] J. Verdasca, M. M. Telo da Gama, A. Nunes, N. R. Bernardino, J. M. Pacheco and M. C. Gomes, J. Theor. Biol. 233, 553 (2005); A. Nunes, M. M. Telo da Gama and M. G. M. Gomes, submitted.

[8] R. M. Anderson and R. M. May, R M, Phil. Trans. R. Soc. Lond. B 314, 533 (1986).

[9] P. Grassberger, Math. Biosci. 63, 157 (1983).

[10] A community of $N$ (fixed) individuals comprises, at time $t, S$ susceptibles, $I$ infectives in circulation and $R$ recovered or removed. Constant infection, $\beta$, and birth/death, $\mu$, rates are assumed. We consider a square lattice of size $N=L^{2}$ with periodic boundary conditions. The (random) variables at each site may take one of the values: $S, I$ or $R$. We account for local connections with $k$ neighbouring sites, with $k=12$, and long-range connections, with a small world probability, $p$. Birth, death and infection occur stochastically, with fixed rates $(\mu, \mu, \beta)$ while recovery is deterministic after $\tau_{i}$ time steps. The recovery time sets the time scale.

[11] In the SEIR model deterministic recovery occurs after $\tau_{l}+\tau_{i}$ steps. Parameters: life expectancy 61 years or $\mu=0.000045$ day $^{-1}$.

[12] From data available at http://www.zoo.cam.ac.uk/zoostaff/grenfell/measles.htm

[13] B. Bolker and B. T. Grenfell, Phil. Trans. R. Soc. London B 348, 309 (1995); B. T. Grenfell, O. N. Bjornstad and J. Kappey, Nature 414, 716 (2001).

[14] M. G. M. Gomes, G. F. Medley and D. J. Nokes, D J, Proc. R. Soc. Lond. B 269, 227 (2002).

[15] L. J. Abu-Raddad and N. F. Ferguson, Proc. R. Soc. Lond. B 271, 2431 (2004); M. F. Boni, J. R. Gog, V. Andreasen and F. B. Christiansen, Theor. Popul. Biol. 65, 179 (2004).

[16] D. Gokaydin, J. B. Oliveira-Martins, I. Gordo and M. G. M. Gomes, submitted.

[17] C. O’ F. Buckee, K. Koelle, M. J. Mustard and S. Gupta, Proceedings of the National Academy of Science 101, 10839 (2004).

[18] T. Nedelea, A. Nunes and M. M. Telo da Gama, work in progress. 


\section{FIGURES}

FIGURE 1 Infectives time series for homogeneously mixed and spatially structured populations from SEIR simulations on $N=1000 \times 1000$ lattices for a) measles $\left(\tau_{i}=8\right.$ days, $\tau_{l}=6$ days $)$ and $\left.\mathrm{b}\right)$ pertussis $\left(\tau_{i}=18\right.$ days, $\tau_{l}=8$ days $)$. a) Grey line: Results for a homogeneously mixed population, $p=1$, and transmission rate $\beta=2.4$ day $^{-1}$. Black line: Results for a network with $p=0.2$ and $\beta=4.75$ day $^{-1}$ also exhibiting an average period close to two years but much larger incidence oscillations. b) From top to bottom: Results for a homogeneously mixed population, $p=1$, and transmission rate $\beta=1.5$ day ${ }^{-1}$; Results for a network with $p=0.2$ and $\beta=4.0$ day $^{-1}$; Results for a network with $p=0.1$ and $\beta=7.0$ day $^{-1}$. As for measles, the transmissibility is tuned to preserve the average period of the homogeneously mixed population. The increase in the incidence peak's amplitude is negligible for $p=0.2$, but for $p=0.1$ the fluctuations are significantly enhanced, as for measles on a network with $p=0.2$.

FIGURE 2 Times series for invasion dynamics simulations of evolved viral strains, on small world networks over lattices with $N=800 \times 800$ nodes. The vertical axis measures the logarithm of the densities of infectives of the circulating and of the invading strains. We have taken 1/80 year ${ }^{-1}$ for the population birth rate, 203 year $^{-1}$ for the transmissibility, 0.27 for the reinfection parameter and 52 year $^{-1}$ for the recovery rate of both diseases. We have considered, in (a), similar strains, with $\delta=0.02$, and, in (b), antigenically distant strains with $\delta=0.2$, and taken $p=1$ (resp. $p=0.3, p=0.1$ ) in the first (resp. second, third) column. 

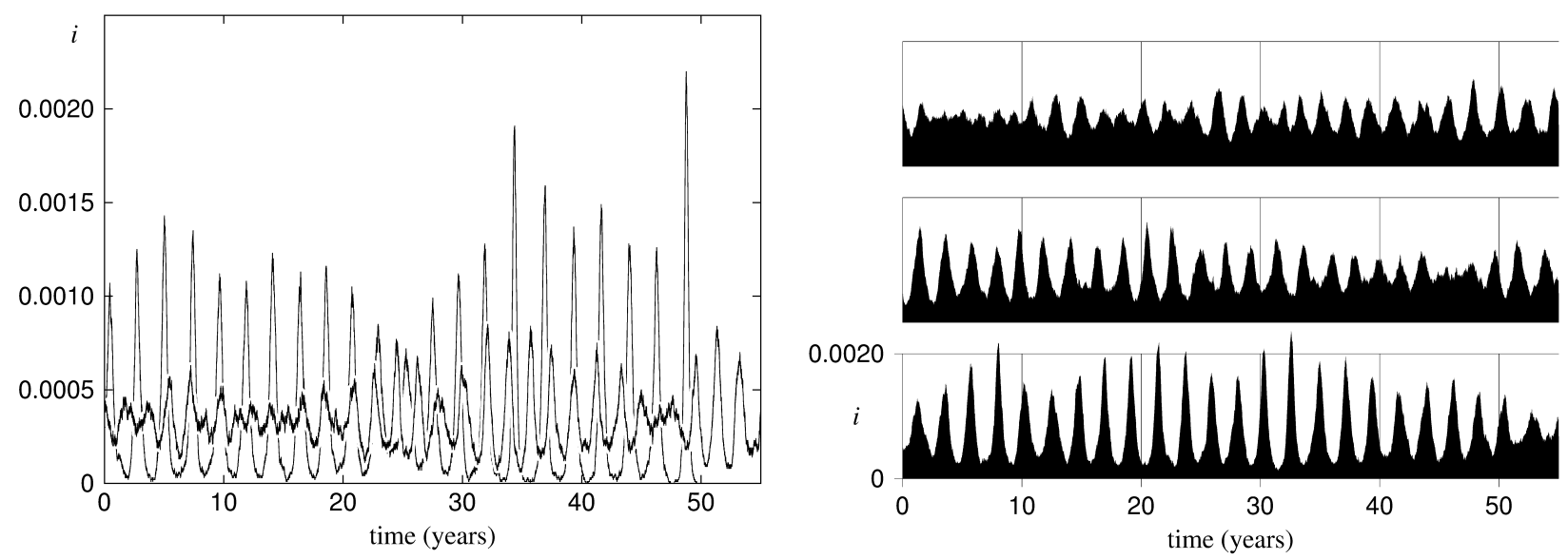

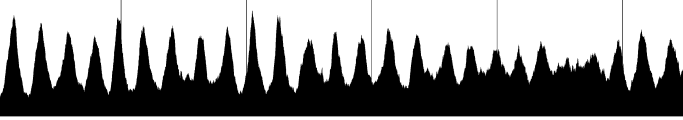

0.0020

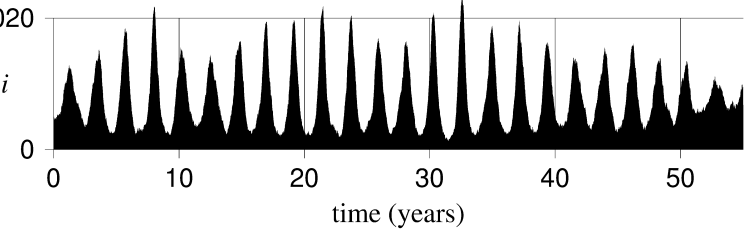

FIG. 1:
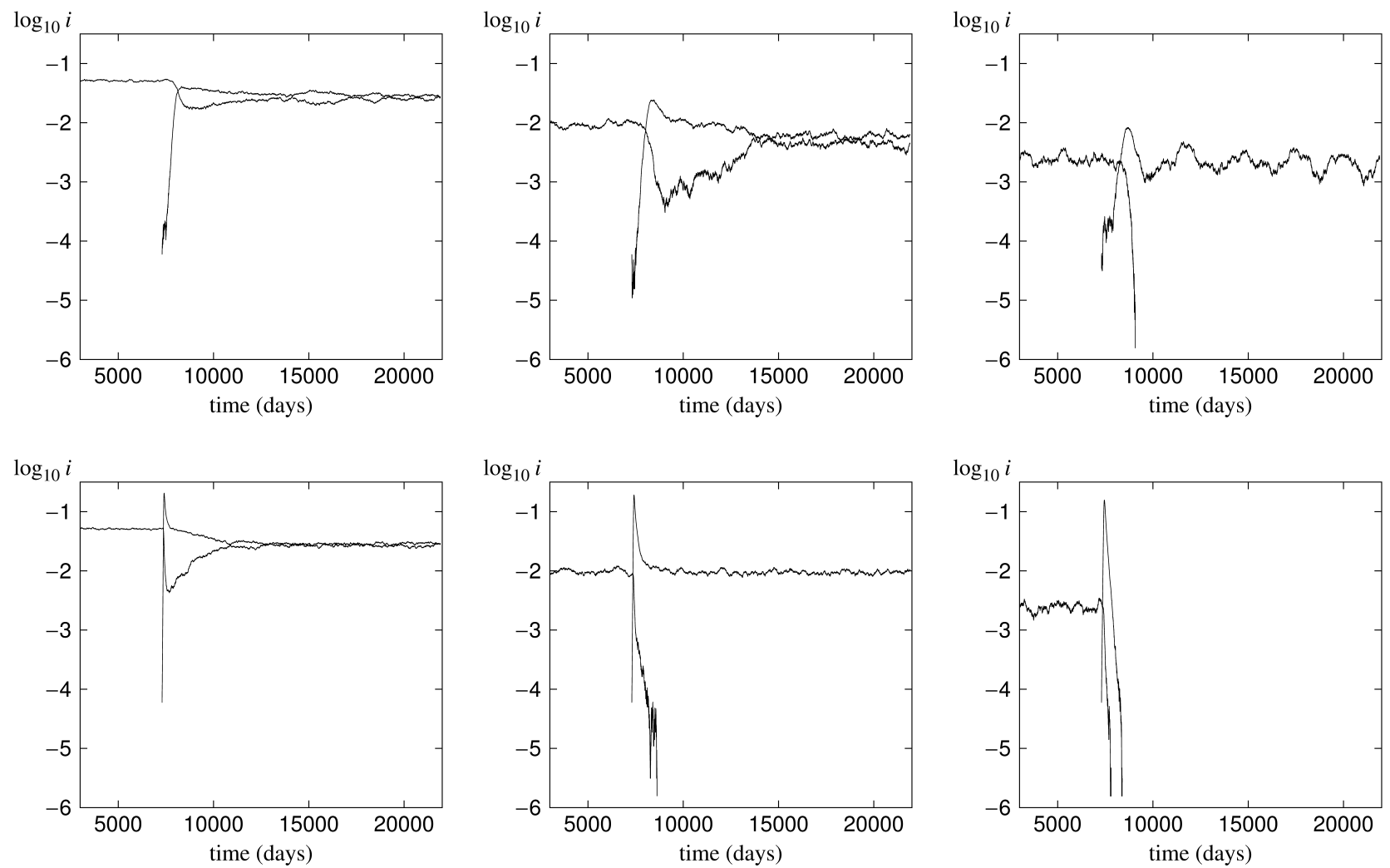

FIG. 2: 